\title{
Prototype for Consultation Cloud loT Supported Medical Records on RFID Technology
}

\author{
Juan Carlos Vesga Ferreira ${ }^{1 *}$, Martha Fabiola Contreras ${ }^{1}$ and Javier E. Sierra ${ }^{2}$ \\ 'Escuela de Ciencias Básicas Tecnología e Ingeniería (ECBTI), Universidad Nacional Abierta y a Distancia; \\ Carrera 27 Nro.40-43.Bucaramanga, Colombia; juan.vesga@unad.edu.co, martha.contreras@unad.edu.co \\ ${ }^{2}$ Facultad de Ingeniería, Universidad de Sucre, Cra 28 \# 5-267, Sincelejo, Colombia;
}

javier.sierra@unisucre.edu.co

\begin{abstract}
Background/Objectives: Telemedicine can be considered IoT evolution of the telecommunications-oriented provision of health services remotely sector. This development implies that it is necessary to have specialized with ability to articulate a consultation service, monitoring or acquisition of information related to the health sector, with ongoing communication processes via Internet devices. The aim of this paper is to design a device capable of consulting the medical history of a patient, which is hosted in the cloud under a scheme of IoT is present. Methods: The prototype is supported under the use of a client / server architecture, which uses an Arduino as processing element, MFRC522 cards MIFIRE operating in the $13.56 \mathrm{MHz}$ band respectively. Results: The results obtained allowed to demonstrate the possibility of developing such solutions at low cost, thereby facilitating its use and overcrowding in all places where this service is required. Application/Improvements: The prototype developed can be considered as a strategy of technological innovation in the field of telemedicine supported in IoT, because that will reveal a fairly quick and efficient medical history of the patient way for any eventuality or routine procedure in order optimize service processes and delivery of services by health agencies. In future related work it is recommended to establish alternative mechanisms of connectivity and storage of information, which allow reaching higher levels of system performance on a large scale.
\end{abstract}

Keywords: Arduino, Medical History, Telemedicine, WiFi, IoT, RFID

\section{Introduction}

RFID (Radio Frequency Identification) is a technology that incorporates the use of electromagnetic or electrostatic coupling in the portion of Radio Frequency (RF) electromagnetic spectrum to uniquely identify an object, animal or person. RFID is widely used in various sectors of industry and telemedicine is no exception. One of the main advantages of RFID technology is that it does not require direct contact or line scan view for the solution you want to develop $\frac{1,2}{\text {. }}$.
An RFID system consists of three components: an antenna, a transceiver (often combined into a reader) and a transponder (tag TAG). The antenna uses radio waves to transmit a signal which activates the transponder. When activated, the tag transmits the data to the antenna. The data is used to notify the programmable logic controller, who is in charge of executing the desired action according to the information recorded in the RFID TAG ${ }^{3}$.

Therefore, an RFID system can be viewed as the sum of the following three components:

${ }^{*}$ Author for correspondence 
1. TAG RFID or transponder

2. Reader or RFID transceiver

3. Subsystem data processing

RFID TAG: In $^{1}$ mention an RFID TAG, it comprises an antenna, a wireless transducer and an encapsulant material is made. These tags can be active or passive. While active tags have on-chip power, passive tags use the power induced by the magnetic field of the RFID reader ${ }^{4}$. Thus, passive tags are cheaper but with a lower range $(<10 \mathrm{mts})$ and more sensitive to regulatory and environmental constraints, compared to active tags $s^{5}$.

RFID reader: An RFID reader comprises an antenna, a transceiver and decoder. The reader emits electromagnetic periodically to detect any RFID tag that may be within their coverage area waves. Upon receiving any signal from a tag, the reader is capable of reading the information contained in the TAG and transmits to the data processor for examination ${ }^{6}$.

Processing System: The data processing subsystem provides the means to process and store information. In most cases, this system can be supported in the use of microcontrollers, which allow the implementation of low-cost embedded systems, aimed at the implementation of specific processing tasks, execution and analysis of information received ${ }^{?}$.

\section{Classification of RFID Systems based on the Frequency}

RFID systems may be classified depending on the frequency range of operation. In ${ }_{-}^{7.8} \mathrm{Y}^{9}$ a description of the classification of RFID systems according blocks frequency range using:

LF RFID: Its scope is in the range of $30 \mathrm{KHz}$ to $300 \mathrm{KHz}$. Most of these devices operate at $125 \mathrm{KHz}$ and $134 \mathrm{KHz}$. The $\mathrm{LF}$ band provides a read range close to $10 \mathrm{~cm}$ and low transmission speeds. Additionally, it is not very sensitive to electromagnetic interference. LF RFID applications including access control and tracking livestock.

The standards for animal tracking systems LF defined in ISO 14223 and ISO / IEC 18000-2. The LF spectrum is not considered a truly global application due to slight differences in the frequency and power levels worldwide.

HF RFID: It's operating range between 3 and $30 \mathrm{MHz}$. Most HF RFID systems operate at $13.56 \mathrm{MHz}$, with read ranges between $10 \mathrm{~cm}$ and $1 \mathrm{~m}$. HF systems experience moderate sensitivity to interference. It is commonly used for applications ticketing, payment and data transfer.

Several HF RFID standards, such as ISO 15693 standard for tracking items and ECMA-340 and ISO / IEC 18092 standards for Near Field Communication (NFC) technology commonly used for short range data exchange between devices. Other standards include HF ISO / IEC 14443 A and ISO / IEC 14443 for MIFARE technology used on smart cards and proximity cards, and the JIS $\mathrm{X}$ 6319-4 for FeliCa, which is a smart card system commonly used in electronic money cards $s^{10}$ due to the benefits of mobility and low-cost implementation. Such networks have been used in common places such as houses, offices, schools among others. The objective of this article is to propose an empiric propagation model, supported in the Log-Normal-Shadowing Path Loss model for $2.4 \mathrm{GHz}$ and $5 \mathrm{GHz}$ bands in Indoor environments, compatible with University campus building. Methods/Statistical Analysis: A scenario of a six-storey building on a university campus was proposed, with dimensions of $60 \mathrm{~m}$ long, $34 \mathrm{~m}$ wide and $24 \mathrm{~m}$ high. Additionally, such building has 24 Access Points (AP).

RFID UHF: Its operating range between $300 \mathrm{MHz}$ and $3 \mathrm{GHz}$. The systems that meet the UHF Gen2 standard RFID uses the band 860-960 MHz. While there is some variation in the frequency of a region other systems UHF Gen2 RFID in most countries between 900 and $915 \mathrm{MHz}$. offer a read range of up to $12 \mathrm{~m}$ UHF systems, transfer rates faster than LF or HF data, but with higher levels of sensitivity to electromagnetic interference. Its applica- 
tions range from inventory management to configuring wireless devices.

The UHF frequency band is regulated by a single global standard called the UHF Gen2 ECP global (ISO 18000-6C) standard.

Classification of RFID Systems based Power System

On the other hand, RFID systems can also be classified according to the power system used by the TAG in assets and liabilities.

Active RFID Systems: In active RFID systems, labels have their own transmitter and power supply. Typically, the power supply is a battery. Active tags emit their own signal to transmit the information stored on their microchips. RFID systems typically operate in the active band Ultra-High Frequency (UHF) and offer a range of up 100 $\mathrm{m}$. In general, active tags are used in large objects, such as cars, large reusable containers and other assets that need to be tracked over long distances ${ }^{11}$.

Passive RFID Systems: In passive RFID systems, the reader sends a radio signal to the tag. The RFID tag then utilizes the signal transmitted to light and reflect the energy back to the reader with the stored information.
The passive RFID systems can operate in radio bands Low Frequency (LF), High Frequency (HF) or Ultra High Frequency (UHF) ranges reading less than $10 \mathrm{~m}$. One advantage of passive tags is that they are cheaper, smaller and easier to manufacture than active tags because not require an additional power supply ${ }^{12}$. It is very important to mention that for prototype development such labels are considered.

Battery-Assisted Passive Systems (BAP): In ${ }^{13}$ It referred to BAP system, which consists of a passive RFID tag battery power. While most of passive RFID tags use the energy of the signal of the RFID reader to power the chip of the label and backscattering the reader, the BAP labels use an integrated power supply (usually a battery) to power the chip. Unlike transponders, the BAP tags do not have their own transmitters.

\section{Prototype Development}

\subsection{Selecting the RFID Tag}

Currently, Philips is the leading manufacturer of tags with MIFARE technology. MIFARE transponders family
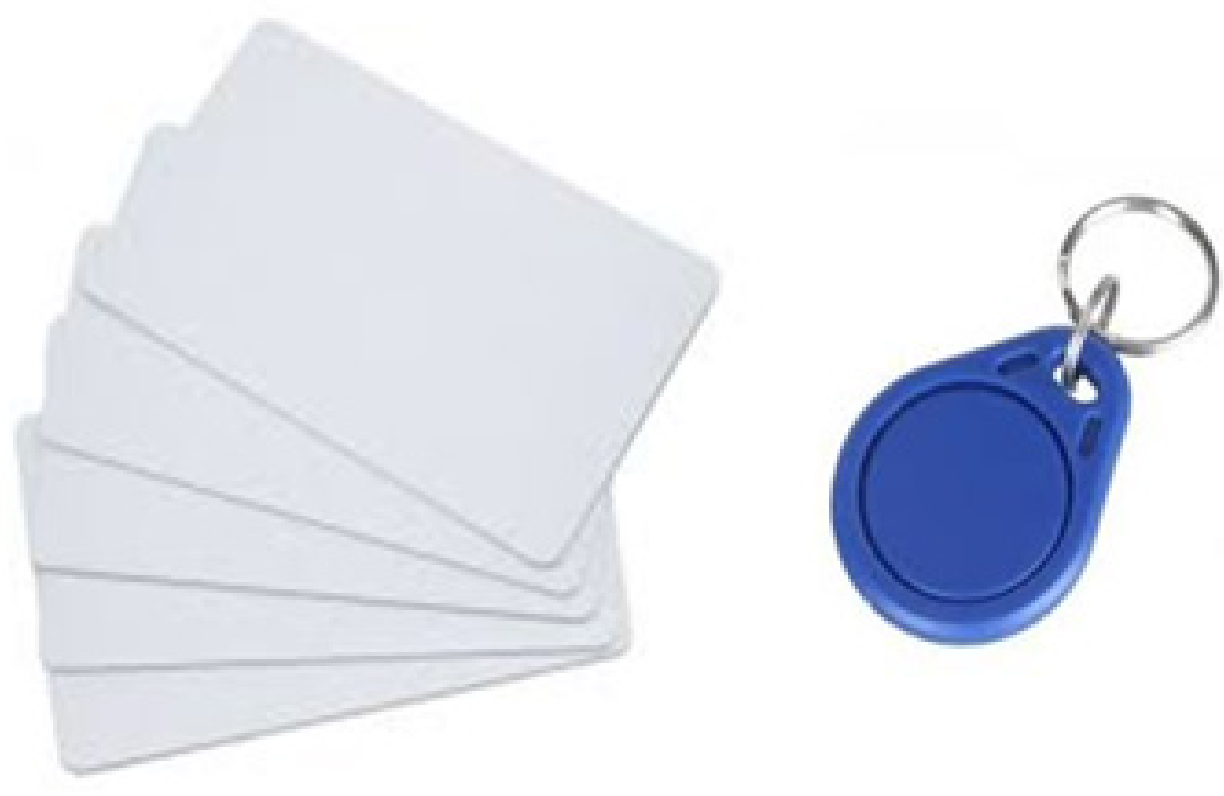

Figure 1. Appearance RFID tag and card type key. 


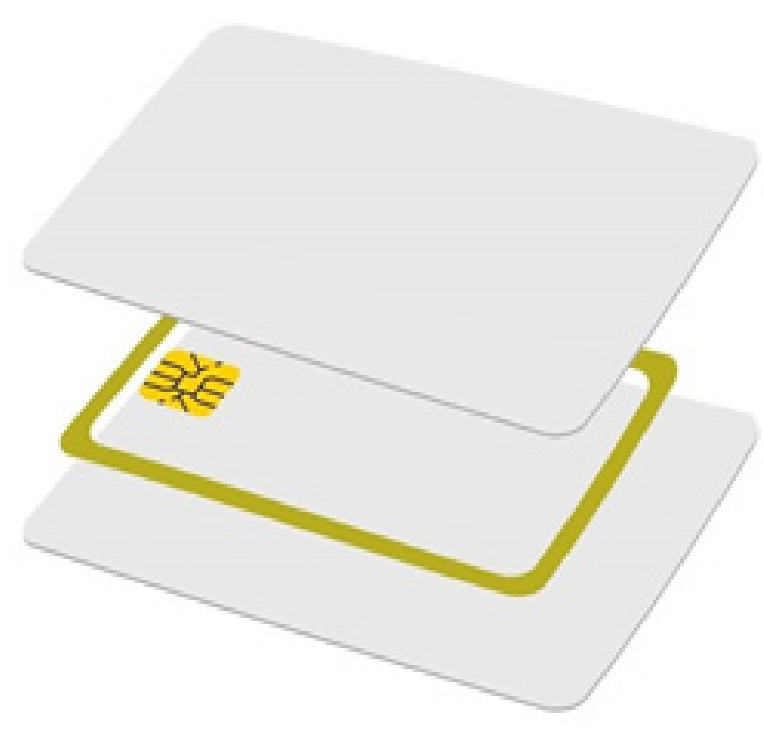

Figure 2. Internal structure of an RFID TAG.

consists of several integrated circuits that operate at a frequency of $13.56 \mathrm{MHz}$, all according to the international standard ISO 14443. This standard is part of the current regulations for the manufacture of electronic identification cards, especially smart cards, jointly run by the International Organization for Standardization (ISO) and International Electrotechnical Commission (IEC) ${ }^{1}$.

The reading distance for this type of TAGs depends on the power of the reader module, which allows on the market, readers of greater and lesser extent. In addition, MIFARE is considered a cheaper and faster technology, why is the most widely used worldwide.

For system development consulting medical records using TAGs type MIFIRE Classic, which can be found commercially in the form of cards or key rings with storage capacities of memory EEPROM $1 \mathrm{~KB}$ and $4 \mathrm{~KB}$ considered. This type of TAG is basically comprised of two electronic elements: a specialized microcontroller known as 'chip' and an antenna for transmission and reception of information at a frequency of $13.56 \mathrm{MHz}$ according to the standard used in the RFID systems $\frac{14}{}$. Figure 1 shows the appearance of the RFID tags that were used to develop the prototype appearance TAGs used in the prototype are presented in Figure 2 and the internal physical structure of the Tag MIFIRE presents illustrated.
As can be seen in Figure 2, within each TAG is a microcontroller, which has an EEPROM memory with read and write, which allows to store the serial number of TAG, known as NUID (Non Unique Identification Number) of 4 bytes which is prerecorded factory, as well as additional information that you want to record on the label. Some TAGs recently manufactured, have a serial number 7 bytes, called UID (Unique Identification Number), which is designed for more complex applications.

This type of TAGs does not have their own power supply. That is, they operate from the electromagnetic field generated by the RFID reader antenna MF522 module to the radio link established. In view of the above, it is necessary to bring the TAG at a distance of approximately 2 to $4 \mathrm{~cm}$ from the RFID module and remain in that position until the read / write EEPROM has finished TAG. During the exchange of data between the devices, use of security mechanisms supported in 16-bit CRC in order to safeguard the integrity of the stored information it is made. The speed of data transmission between the TAG and the RFID device reaches about $100 \mathrm{KBITS} / \mathrm{sec}^{\frac{15}{5}}$.

The functional description of MIFARE Classic TAG used for consulting medical records is as follows:

- MIFARE Classic 1K - Contactless Smart Card

- It has the corresponding MF1S503x chip to a $1 \mathrm{kB}$ EEPROM, an RF interface unit and a digital control.

- The power and data are transferred through an antenna consisting of a coil with a small number of turns is connected directly to MF1S503x. No external components are needed most.

- RF interface:

- Modulator/Demodulator

- Rectifier

- Regenerating clock

- Power-on reset (POR)

- Voltage regulator 
- Anticollision: several cards in the field may be selected and operated in sequence

- Authentication: The authentication procedure ensures that access to a block is only possible through the two keys specified for each block

- Control unit and arithmetic logic: The values are stored in a special redundant format and can increase and decrease

- Encryption Unit Crypto1 the MF1S503x used for authentication and encryption of data exchange.
- EEPROM: $1 \mathrm{kB}$ is organized in 16 sectors with 4 blocks each. A block contains 16 bytes. The last block of each sector is called "trailer", which contains two secret keys and programmable access conditions for each block in this sector.

\subsection{Memory Organization EEPROM 1Kb TAG}

In $\frac{16}{}$ the memory organization for TAG described with an EEPROM of 1024 bytes. However, because of the locations used for security codes, the number of useful sites the user for data storage is 768 bytes. It is organized in 16
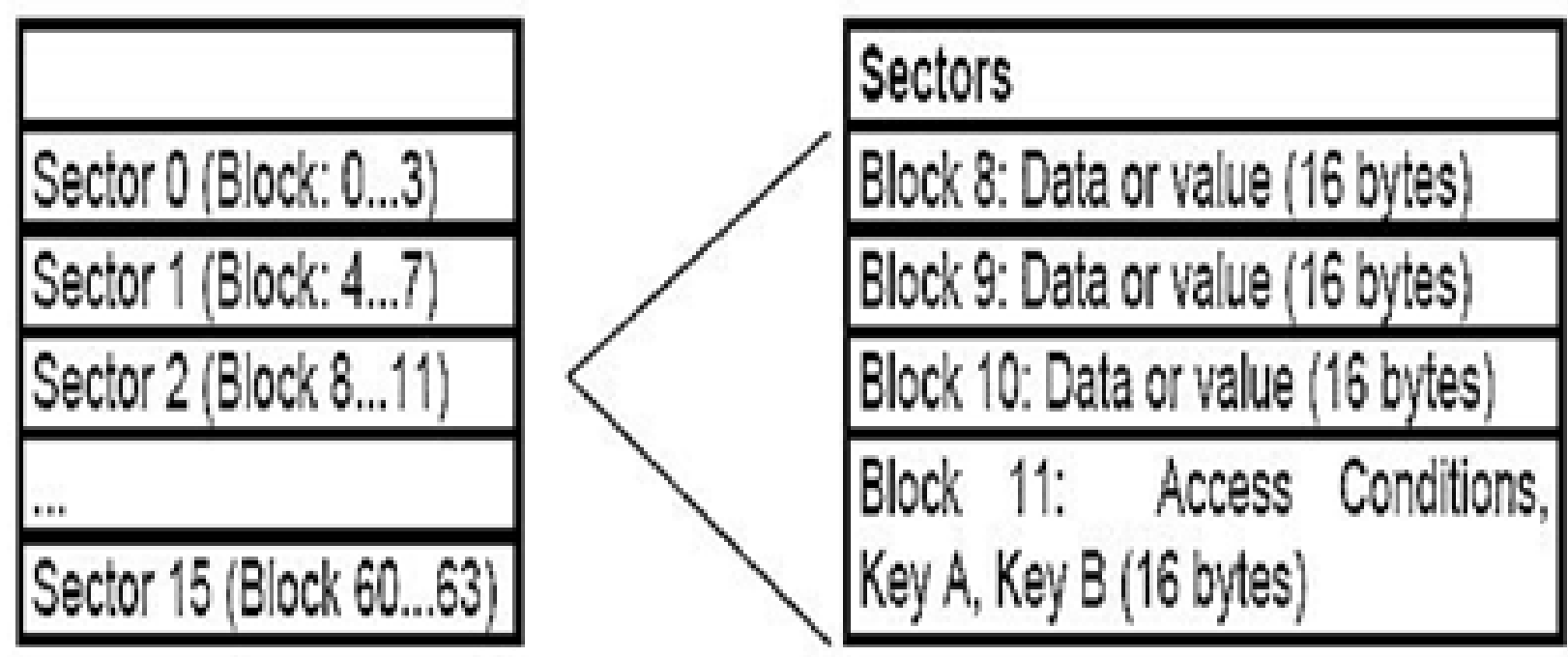

Figure 3. Schematic organization EEPROM memory.

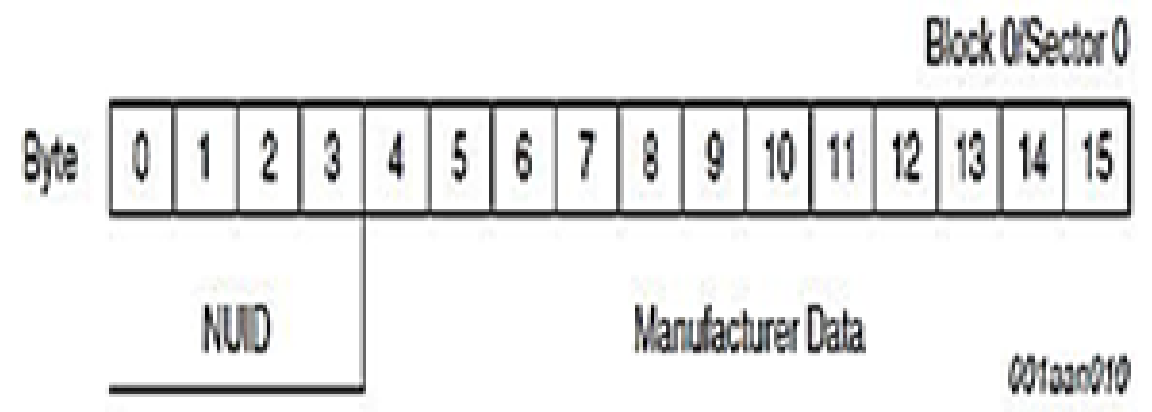

Figure 4. Internal structure of sectors and blocks EEPROM memory. 
sectors of 4 blocks each, for a total of 64 blocks. In turn, each block is constituted by 16 bytes. In Figure 3 a diagram of the memory organization of TAG occurs.

Figure 4 presents the structure of internal sectors and blocks that are part of the EEPROM. It shows that II first 4 bytes of sector 0 of block 0 ; store the serial number of the TAG (NUID) and manufacturer information, which is unique to each tag. This section is protected and only allows reading processes will be used by the system to identify whether the label is among the valid tags, in order to provide a first level of security against possible sources of impersonation ${ }^{12}$.
A second security mechanism used is the use of the fourth block of each sector known as "Trailer Sector", which lets you set permissions and access codes for reading / writing data from that sector. To make the process of reading and writing information in each block is needed with 2 security codes called Key A and B. Key A can be written but not read. Furthermore, the key B is optional and may be used or not by the system programmer. The default values for both keys Key A and Key B correspond to 0xFFFFFFFFFFFF and "Access bits" have values 0xFF 0x07 0x80 0x69 $9^{17}$. Figure 5 shows the structure of a trailer industry.

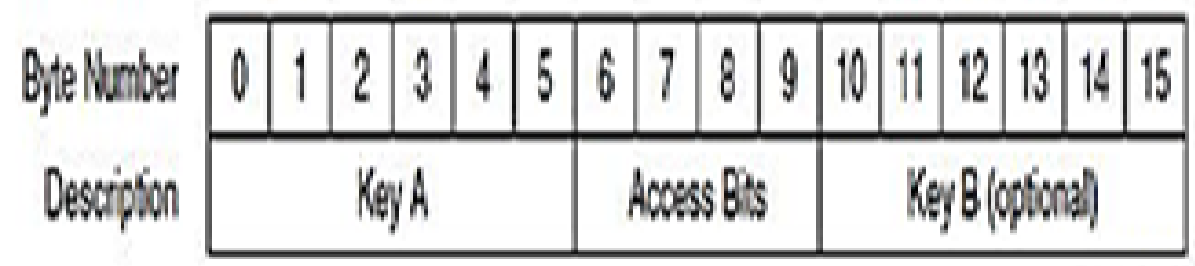

Colowis

Figure 5. Structure sectors TAGs RFID.

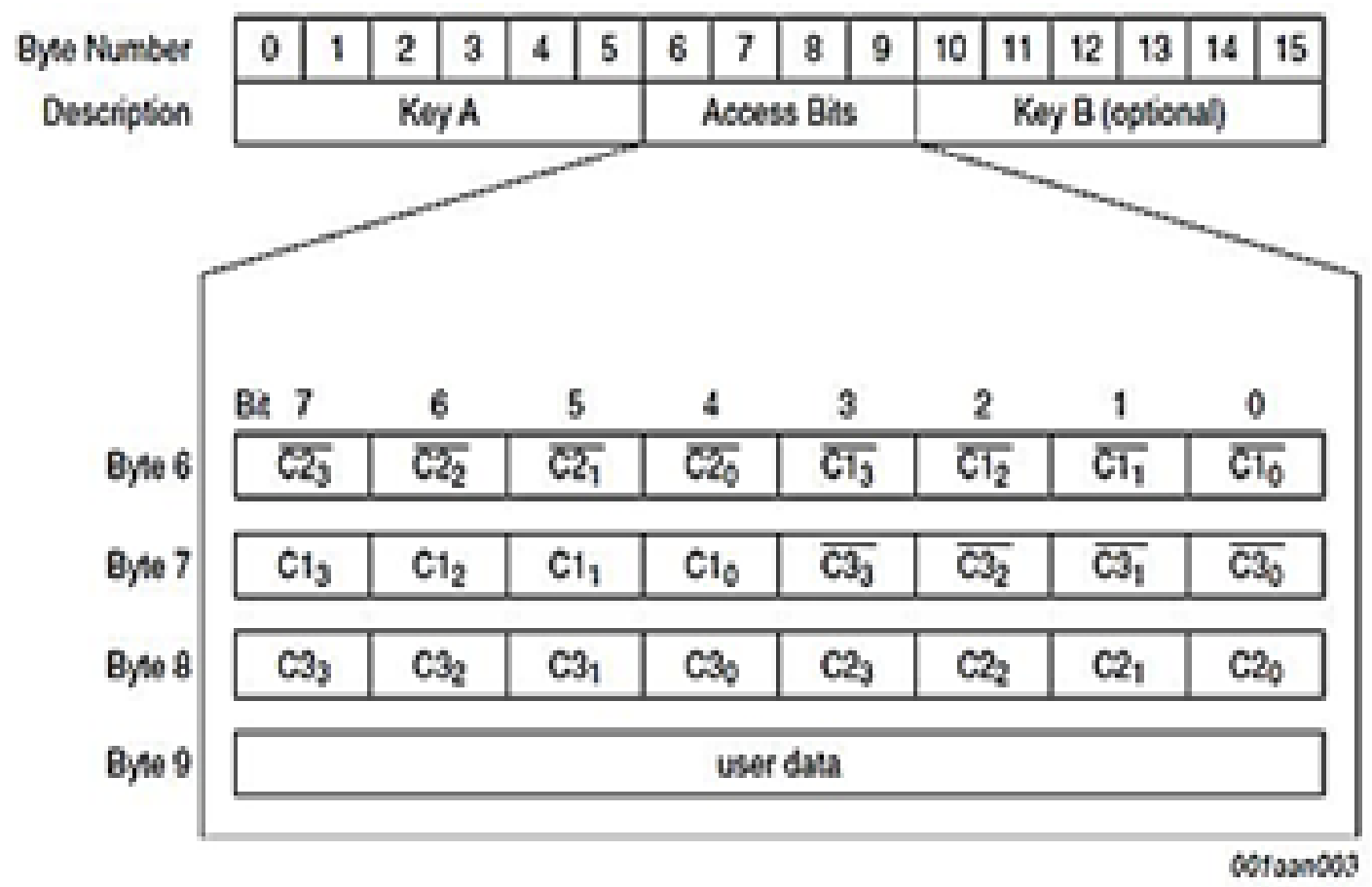

Figure 6. Structure access bits in TAGs RFID. 
The TAG $1 \mathrm{~Kb}$ has 16 "Trailers Sector", identified with the numbers $3,7,11,15,19,23,27,31,35,39,43,47,51$, 55,59 and 63 , which they can only be used to establish the credentials of access to information of each block.

Finally, Figure 6 gives the structure of so-called "Access bit", which correspond to a group of 3 bits, C1, C2 and C3, which define permissions for each of the four blocks of the sector is presented. In the following scheme 6 bits occurs access $\frac{18}{}$.

\section{Reader Module/Written RFID MFRC522}

The RFID reader module is based on the integrated MFRC522 NXP who specializes in "contactless communication" IC, which supports ISO / IEC 14443 A or MIFARE tag circuit. The module includes an antenna for read / write with all necessary to communicate with the TAGs architecture. In view of the above, the user must connect the module only by the SPI interface Arduino, which serves as control module system ${ }^{14}$. In Figure 7, the physical appearance of MFRC522 module, used for reading / writing of RFID Tag, during development of the prototype is presented.
Next, the technical specifications of MFRC522 module:

- Model: MF522-AN, read-write data, based on the MFRC522 Philips chip. It Includes 2 tags and key type of card.

- approximate detection distance $=5 \mathrm{~cm}$.

- Voltage: 3.3 volts @ 13 ma. Maximum current = $30 \mathrm{~mA}$.

- Tag specification: ISO-14443 MF1S503, $1 \mathrm{~Kb}$ EEPROM

- Operating Frequency: $13.56 \mathrm{Mhz}$

- Interface: header connector 8 pin, SPI, with speeds up to $10 \mathrm{Mbps}$.

- Operating Temperature: -20 to +60 degrees C.

- Dimensions: 40 x $60 \mathrm{~mm}$.

- TAGs allows reading and key card type.

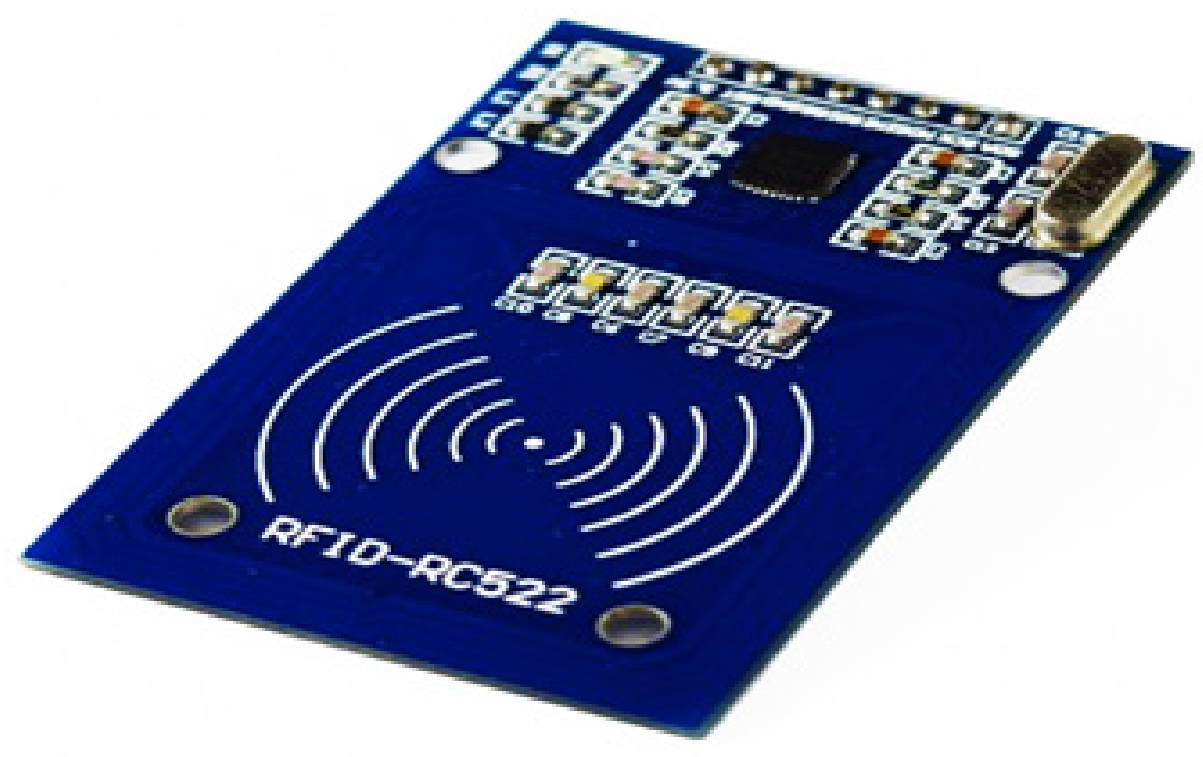

Figure 7. The module for reading RFID MFRC522. 


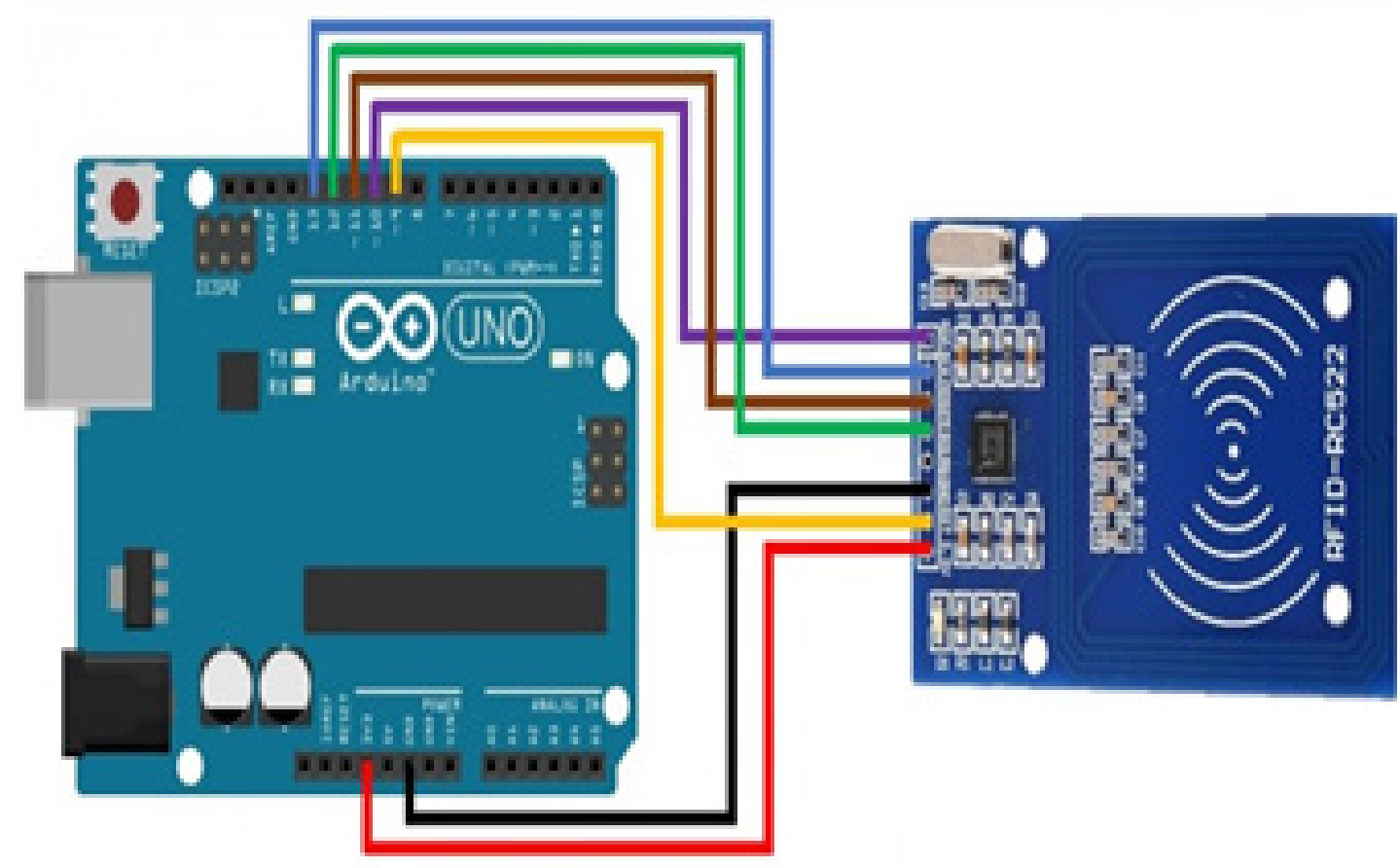

Figure 8. Connection diagram of RFID module - Arduino.

Table 1. PinOut of connection between the RFID module and Arduino

\begin{tabular}{|c|c|c|}
\hline RC522 module & Arduino Uno, Nano & Arduino Mega \\
\hline SDA (SS) & 10 & 53 \\
\hline SCK & 13 & 52 \\
\hline MOSI & eleven & fifty \\
\hline MISO & 12 & Not connected \\
\hline IRQ & Not connected & GND \\
\hline GND & GND & 9 \\
\hline RST & 9 & $3.3 \mathrm{~V}$ \\
\hline $3.3 \mathrm{~V}$ & $3.3 \mathrm{~V}$ & \\
\hline
\end{tabular}

Figure 8 shows the connection scheme between the processing system based on Arduino platform and MF522 module is illustrated.

Table 1 shows the pinout connecting the RC522 and Arduino relay. As noted, the module works with a volt- age of $3.3 \mathrm{~V}$, an aspect that should be considered of vital importance in energizing the circuit to avoid destroying error, some of the components forming part of the system. In view of the above, the use of converters of voltage levels is recommended. 


\section{Programming Module RC522: Reading the Identification Code}

Module RC522 to work under Arduino platform is necessary to use RFID library, developed by Miguel Balboa. Once downloaded, it is necessary to carry out the process of loading and updating the IDE to initiate the programming process $\frac{19}{}$.

Then a skit shown for reading the identification code of our Tags

\#include $<$ SPI. h $>$

\#include $<$ MFRC522.h>

\#define RST_PIN 9 // Pin 9 for the reset RC522

\#define SS_PIN 10 // for SS Pin 10 (SDA) of the RC522

MFRC522 MFRC 522 (SS_PIN, RST_PIN); // Create

object for RC522

void setup () \{

Serial. begin (9600); // Starts serial communication

SPI. begin (); // Start Bus SPI

MFRC 522.PCD_Init (); // Start the MFRC522

Serial. println (“Reading the UID”); \}

Void loop () \{// Revision for new cards present

if (mfrc522.PICC_IsNewCardPresent ())

\{

// Select a card

if (mfrc522.PICC_ReadCardSerial ())

\{

// We send serialemente your UID

Serial.print ("UID Card");

for (byte $\mathrm{i}=0$; $\mathrm{i}<\mathrm{mfrc522.uid.size;} \mathrm{i}++$ ) \{
Serial. Print (mfrc522.uid.uidByte [i] <0x10 “0”: “");

Serial. Print (mfrc522.uid.uidByte [i], HEX);

\}

Serial.println ();

// Terminate reading the current card

mfrc522.PICC_HaltA ();

\}

\}

\}

In the code base it is necessary to use the MFRC522.h library, which contains the basic configuration parameters and definition of special functions for using the RC522 module.

Start the RC522

SPI. begin (); // we begin the SPI Bus

mfrc522.PCD_Init (); // we begin the MFRC522

The SPI.begin function starts and configures the RC522 for later reading. You only need to invoke once since section void setup ().

To assess whether card is present, use mfrc522.PICC_ IsNewCardPresent (function blocks) which returns as result "true" or "false" depending on whether detection of a card present near the module RC522.

To make the process of reading an RFID card use mfrc522.PICC_ReadCardSerial () function, which returns true if he can select a card reading, or false otherwise done.

To access the identification code mfrc522.uid.uidByte use of the function is done, it is advisable to index in a variable. For example, mfrc522.uid.uidByte [0] for byte in the initial position.

To complete the process of reading use mfrc522. PICC_HaltA function () it is made, through which we 
indicate the system has finished reading this card. If after removing the card, you enter the same card again is regarded as a new card.

\section{WiFi Module by ESP8266}

To establish communication processes between the terminal and the application RFID Web using TCP / IP protocol, it is necessary to implement a wireless communication module, which will allow access to the database through WiFi network. For this, the RFID terminal uses the ESP8266 module (Figure 9), which allows you to communicate over the Internet in a wireless manner, facilitating aspects of mobility, ubiquity and portability of the prototype developed.

ESP8266 WiFi Module is a stand-alone SOC stack TCP / IP protocol that can provide integrated access to any microcontroller to your WiFi network. The ESP8266 is able to accommodate an application or download functions WiFi network from another application processor. ESP8266 Each module comes preprogrammed with firmware AT command set, which means you can simply connect to the Arduino platform and get almost WiFi capability offers Wi-Shield. The ESP8266 module is low cost and ease of programming and joint systems requiring establish communication processes via the Internet or some network infrastructure wirelessly ${ }^{20}$.

This module has a storage capacity and sufficiently robust processing to implement complex and capable of articulation with sensors and other devices through its GPIOs solutions. Its high degree of integration on the chip allows minimal external circuitry, including frontend module is designed to occupy a minimum area of PCB. The ESP8266 supports APSD and interfaces for VoIP applications Bluetooth coexistence. RF system additionally contains self-calibrated, which allows working in all operating conditions without additional communication elements ${ }^{21}$.

Figure 10 presents the connection diagram ESP8266 Arduino platform module.

Among the main technical characteristics of ESP82266 module may be mentioned:

- $802.11 \mathrm{~b} / \mathrm{g} / \mathrm{n}$

- Wi-Fi Direct (P2P), soft-AP

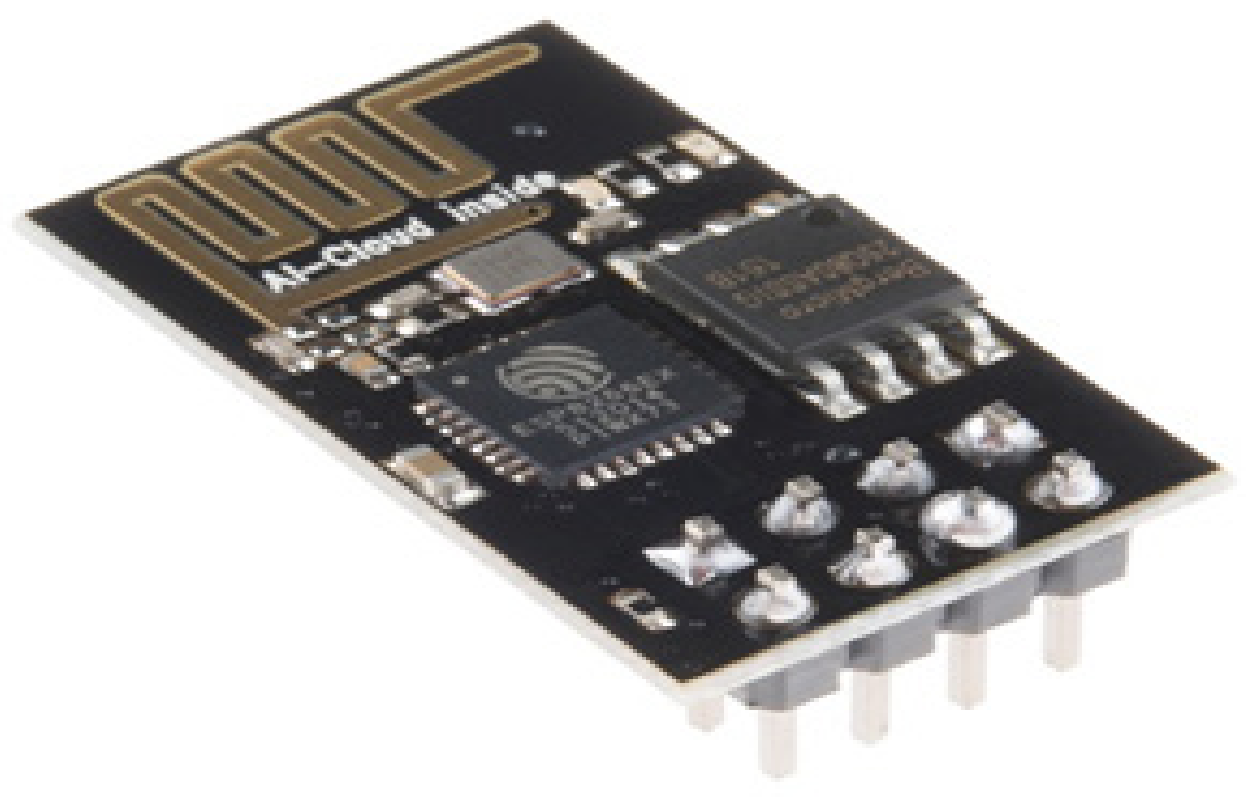

Figure 9. Module ESP8266. 


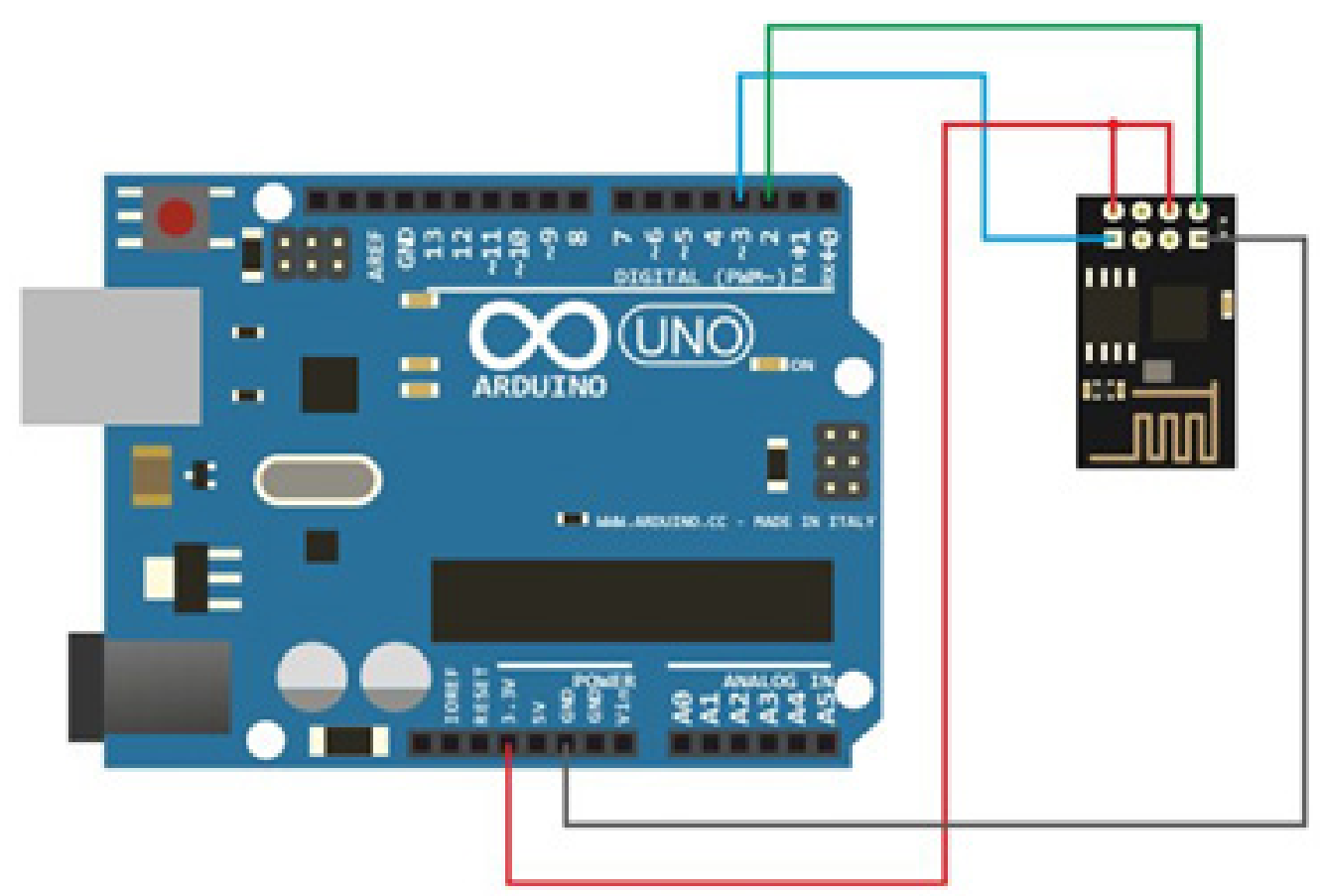

Figure 10. Wiring Arduino - ESP8266.

- Stack TCP / IP protocols integrated

- TR switch integrated balun, LNA, power amplifier and matching network

- integrated PLLs, regulators, DCXO and power management units

- $\quad+19.5 \mathrm{dBm}$ output power in $802.11 \mathrm{~b}$ mode

- Leakage current $<10 \mathrm{uA}$

- $1 \mathrm{MB}$ flash memory

- CPU 32-bit low-power could be used as integrated application processor

- $\quad$ SDIO 1.1 / 2.0, SPI, UART
- $\quad$ STBC, 1 x 1 MIMO, MIMO 2 x 1

- A-MPDU \& A-MSDU aggregation \& $0.4 \mathrm{~ms}$ guard interval

- Awakes and transmits packets in $<2 \mathrm{~ms}$

- Standby power consumption of $<1.0 \mathrm{~mW}$ (DTIM3)

\section{TFT LCD Touch for Arduino 2.4 "}

In order to establish a visual communication interface between the user, the RFID terminal and Web system using a touch LCD 2.4 "screen is done, which can be articulated with the Arduino platform, allowing display information sent from the web application related to the RFID user ${ }^{22}$. Figure 11 presents a physical appearance of the Touch TFT 2.4 "screen. 


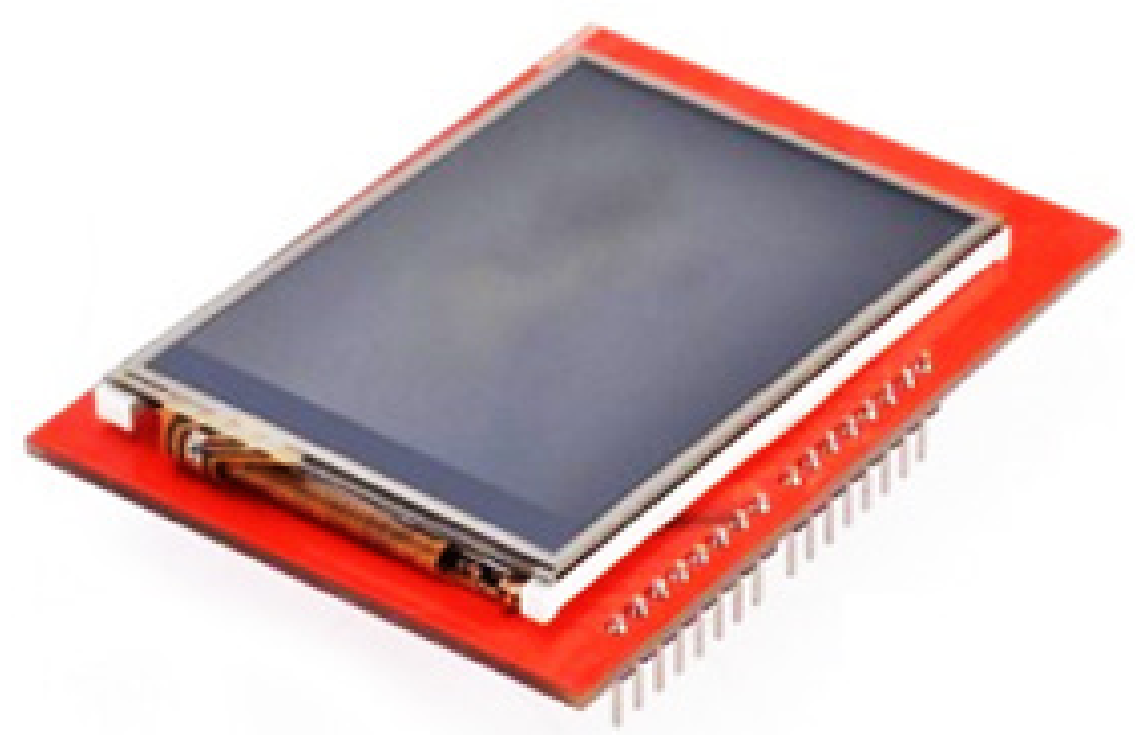

Figure 11. Touch Screen TFT 2.4".

Table 2. Technical specifications TFT screen

\begin{tabular}{|c|c|}
\hline Kind & TFT \\
\hline digital interface & 8 bits (four control lines) \\
\hline Touch Pad & SPFD5408 (video RAM) \\
\hline Supply voltage & $5 \mathrm{~V}$ (3.3V logic levels) \\
\hline Operating Current & $300 \mathrm{~mA}$ \\
\hline type storage & micro SD \\
\hline Colors & $65 \mathrm{~K}$ \\
\hline driver & ST7781 \\
\hline digital pins & $5-13$ \\
\hline analog pins & $0-3$ \\
\hline inches & 2.4 inches \\
\hline Resolution & 240px X 320px \\
\hline Color range & 18 bits (262 000 tones) \\
\hline
\end{tabular}

This TFT LCD is designed to be used under Arduino platform for its pinout is also compatible with the Duemilanove and Diecimila (not compatible with Arduino Mega or Leonardo). Additionally, this screen is 


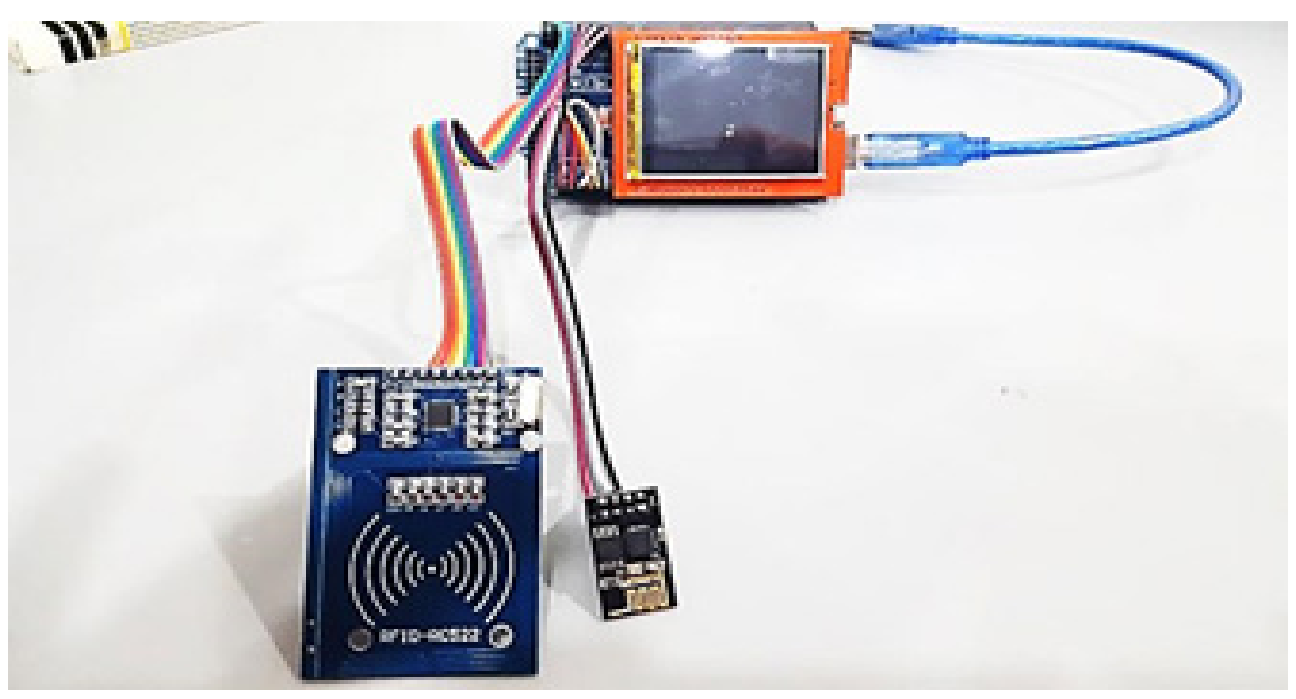

Figure 12. Appearance Terminal RFID.

touch, which allows you to receive information tactilely detecting the finger pressure in any area of the LCD. The TFT LCD has a size of 2.4 inches, with a resolution of 240px X 320px, bright LED backlight (varies if connects a transistor) with a palette wide (18 bits, up to 262,000 different tones). Table 2 shows the specifications of the TFT that was used during the development of the prototype are presented.

Among the main technical specifications of the screen can be mentioned:
Figure 12 shows the resulting physical appearance of the prototype for consultation cloud based on the use of RFID technology presents case histories.

\section{Description of Medical Records System under loT Architecture}

Various applications running on a client/server environment. This means that client computers (in this case the terminal prototype RFID) seek to establish communica-

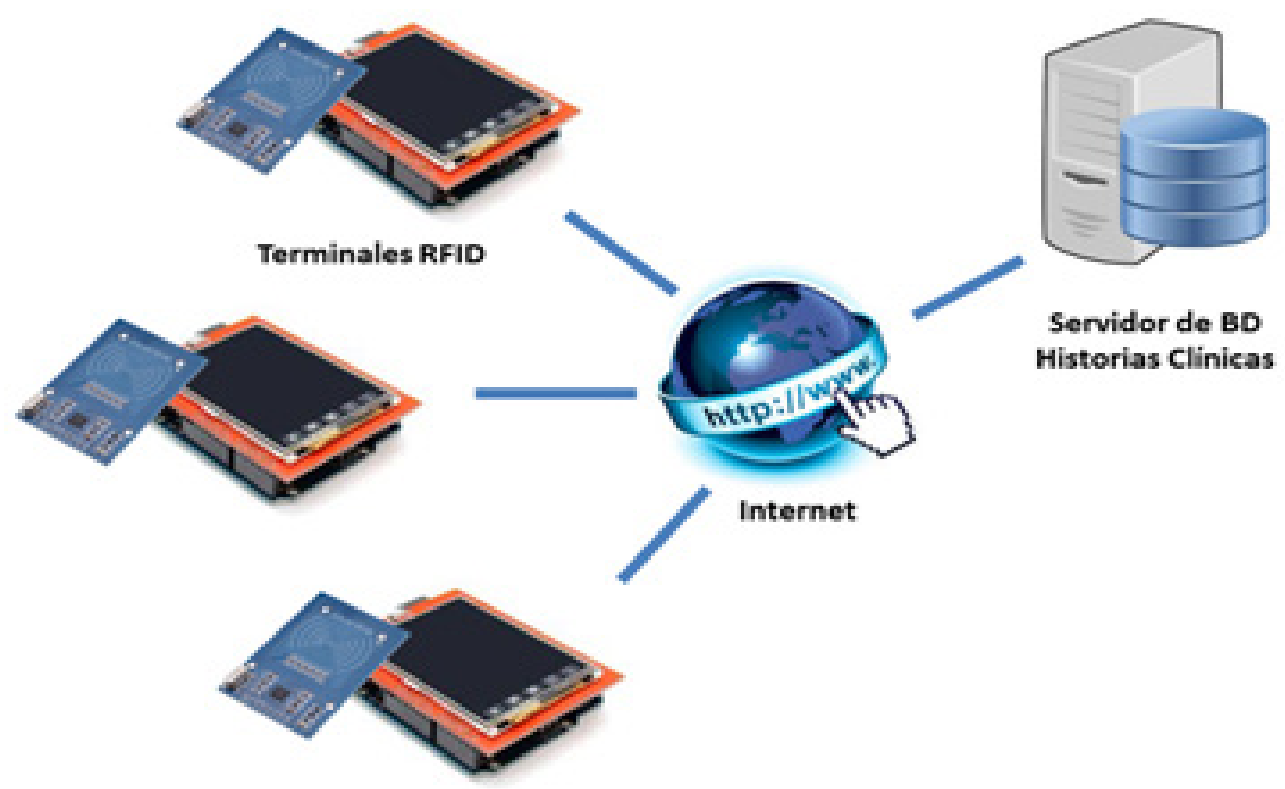

Figure 13. Client-Server Architecture for the proposed system. 


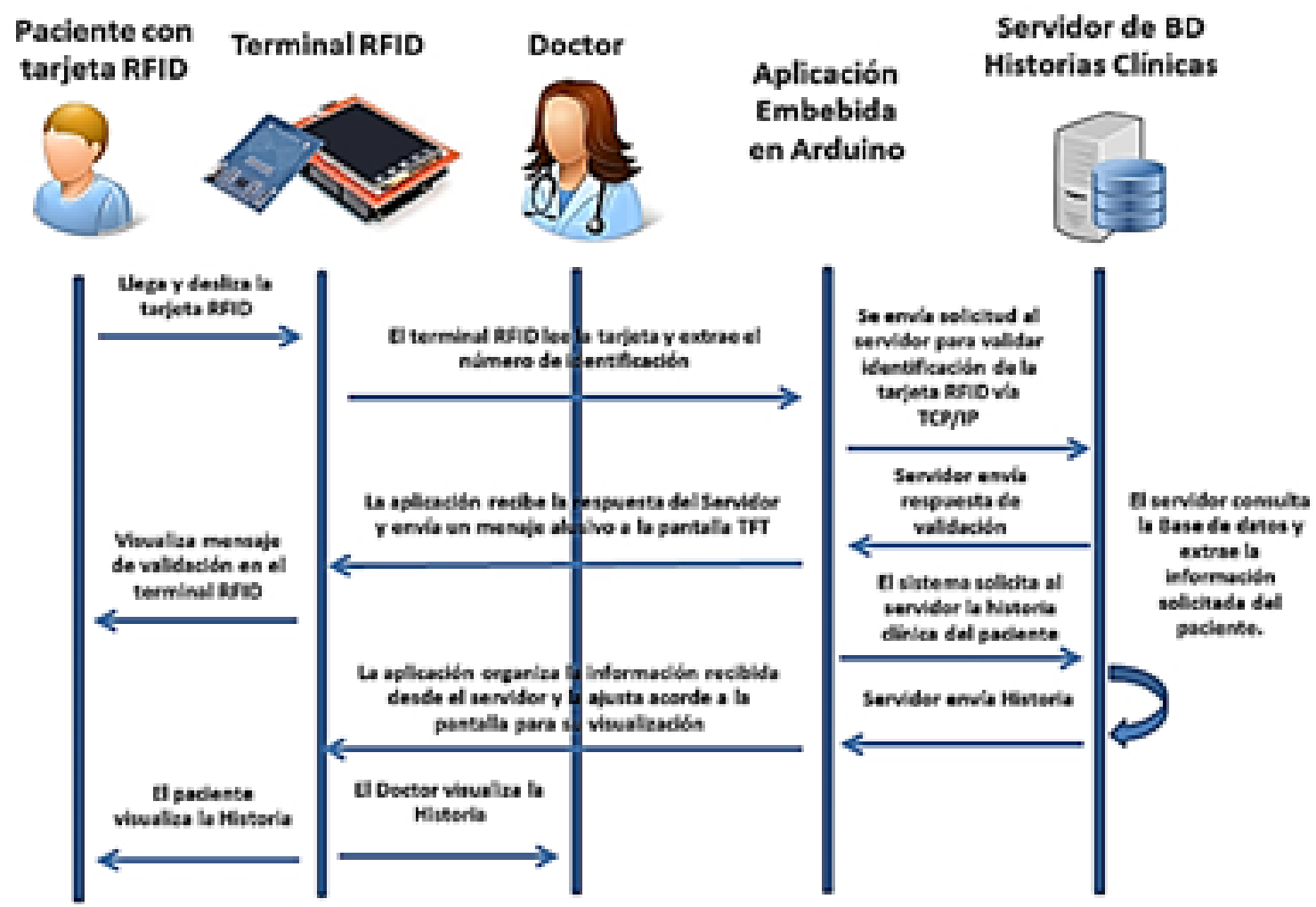

Figure 14. Sequence Diagram - System consulting medical records through RFID.

tion with the server, from which all information related to medical records is. The proposed system makes use of this type of architecture, where RFID terminals can be located anywhere and through internet, send query requests to the main server, which, according to the number of ID card and number of medical history, performs the consultation process in the database, making the latter, sending information under web environment back to the respective RFID terminal. Figure 13 clearly illustrates the client-server architecture for the proposed system.

As mentioned above, the prototype uses the RC522 module as RFID reader / writer and ESP8266 module as a strategy for communication processes using TCP / IP over 802.11 protocols or WiFi. Figure 14 is the system interaction diagram for consulting medical records, where all processes requests that are linked during the reading process, and query information display described is presented.
For the registration process and information storage server database, supported in MySQL through which the relationship for each of the tables and fields that are part of medical records system designed entity model was implemented.

The database consists of three tables where the general data are stored, the objective and subjective patient information. In order to maintain data integrity between tables the number of clinical history as a main course or main key association information between tables is established, reducing redundancy and duplication issues information $^{23}$. Figure 15 provides the proposed structure of the database is presented.

The main advantages of client-server architecture can be mentioned ${ }^{2} 4$ :

- The system has centralized resources, because the server is the central system and therefore it is pos- 


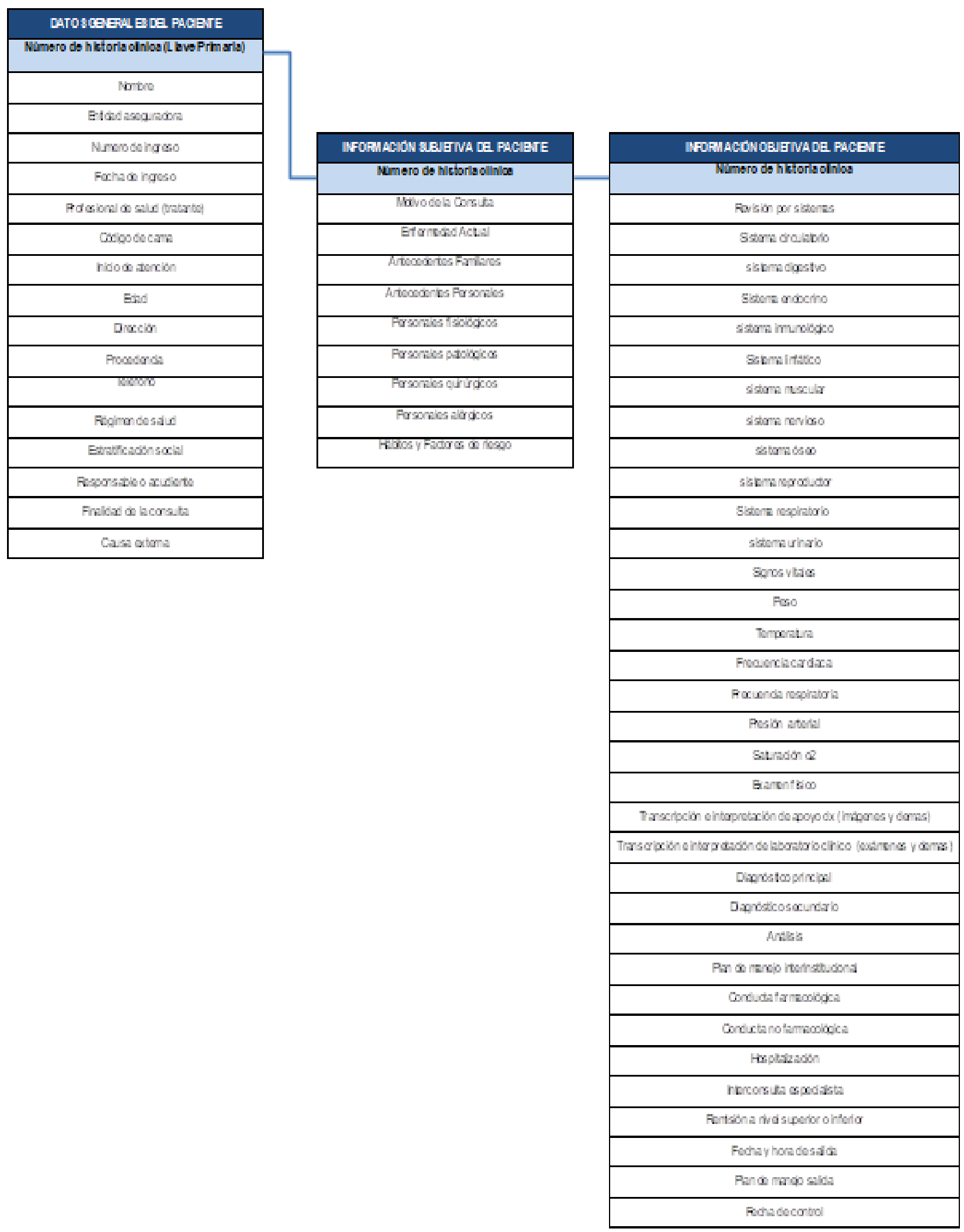

Figure 15. Structure of the proposed database for managing medical records. 


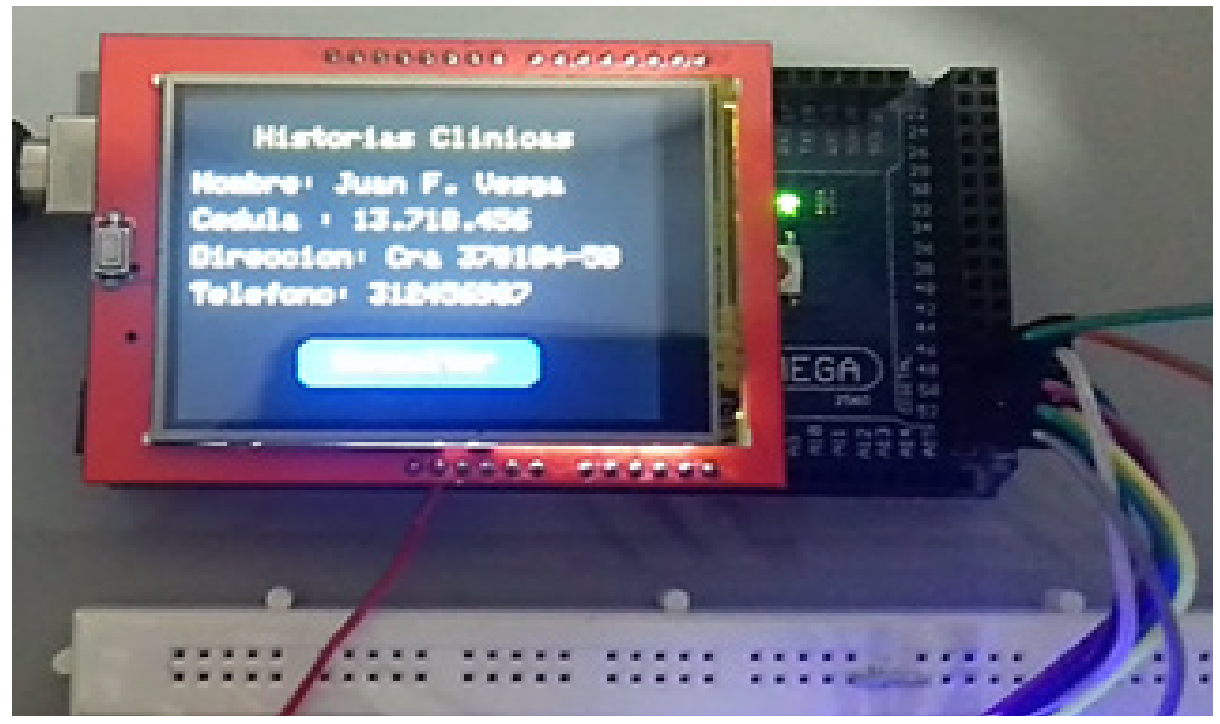

Figure 16. Result of a consultation process of the clinical history of a patient recorded.

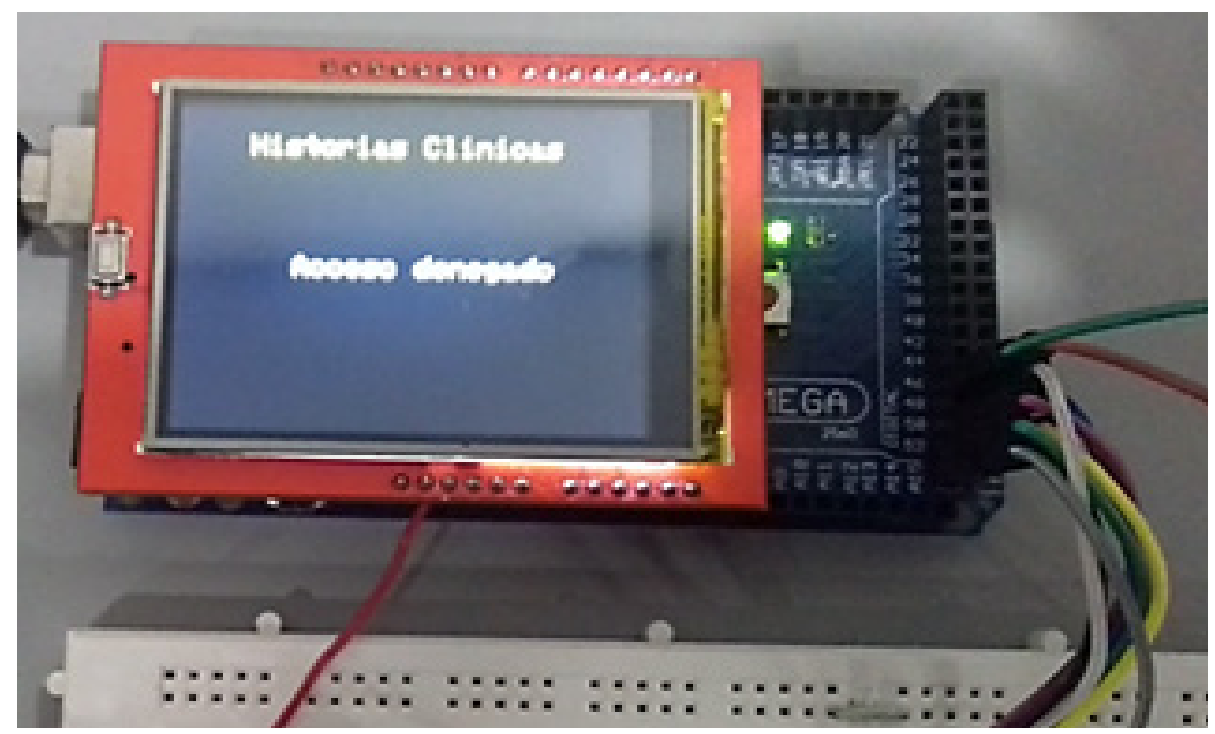

Figure 17. Result of a consultation process medical history of an unregistered user or lacking a valid card to the system.

sible to manage resources that are common to all users.

- High levels of security and availability

- It requires lower levels of administration and configuration
- Sets escalation processes and continuous growth.

- Accept the requirements of databases that make customers.

- Process the application logic and validations level databases 
Figure 16 shows the results obtained by making use of an RFID tag of a patient, where you can see the personal data and review of medical history which was queried cloud is presented. Furthermore, Figure 17 shows on the display the result obtained after trying to use an RFID tag that has not been registered in the system and thereby validates the situation where a patient wants to use a card which it is invalid by the system.

\section{Conclusions}

The use of RFID technology can be considered an excellent alternative in developing Cloud-based Computing for managing information in the field of health systems. During the development of the proposed prototype it was evident that it is possible to design stable systems and quite robust, with WiFi connectivity as a means of communication with the centralized database in the cloud and supported in the use of TAG Mifire such as mechanism user identification for consultation of medical records. The proposed system not only demonstrated a level of functionality and quite high efficiency, but also reflects a clear feasibility of being implemented at very low cost in various hospitals requiring to have this service.

\section{References}

1. Want R. An introduction to RFID technology. IEEE pervasive Computing. 2006; 5(1):25-33. https://doi.org/10.1109/ MPRV.2006.2.

2. Vesga Ferreira JC, Antonio Vesga Barrera J, Sierra JE. Design of a prototype remote medical monitoring system for measuring blood pressure and glucose measurement. Indian Journal Science and Technology. 2018; 11(22):1-8. https:// doi.org/10.17485/ijst/2018/v11i20/122509.

3. Bhuptani M, Moradpour S. RFID field guide: Deploying radio frequencyidentification systems. Higher Education, Prentice Hall; 2005. PMid:15920803. PMCid:PMC1963916

4. Sierra JE, Carlos Vesga J, Medina B. Livestock model innovation under the concept of smart cities. Indian Journal Science and Technology. 2018; 11(22):1-6. https://doi. org/10.17485/ijst/2018/v11i22/122510.

5. Landt J. The history of RFID. IEEE Potentials. 2005; 24(4):811. https://doi.org/10.1109/MP.2005.1549751.
6. Ni L, Liu Y, Lau Y, Patil A. LANDMARC: indoor location sensing using active RFID. Proceedings of the First IEEE International Conference on Pervasive Computing and Communications; 2003. p. 407-15. https://doi.org/10.1109/ PERCOM.2003.1192765.

7. Dobkin D. The RF in RFID: UHF RFID in Practice. Newnes; 2012. p. 1-529.

8. Fu Y, Qian Z, Wang X, Liu G. Identifying the unknown tags in a large RFID system. China Communications. 2017; 14(1):135-45. https://doi.org/10.1109/CC.2017.7839764

9. Nikitin P, Rao K. Antennas and propagation in UHF RFID systems. IEEE International Conference on RFID; 2008. 277-88.

10. Carlos Vesga FJ, Martha Fabiola Contreras H, Jose Antonio Vesga B. Design of empirical propagation models supported in the Log-Normal Shadowing model for the $2.4 \mathrm{GHz}$ and $5 \mathrm{GHz}$ bands under Indoor environments. Indian Journal Science and Technology. 2018; 11(22):1-18. https://doi. org/10.17485/ijst/2018/v11i20/122149.

11. Nikitin P, Rao K, Martinez R. Sensitivity and impedance measurements of UHF RFID chips. IEEE Transactions on Microwave Theory and Techniques. 2017; 57(5):1297-302. https://doi.org/10.1109/TMTT.2009.2017297.

12. Ye Y, Ru Y, Liu D, Huang J. Study on inbound special goods traceability system on the basis of RFID technology. IEEE International Conference on Logistics, Informatics and Service Sciences (LISS); 2016. p. 1-6. https://doi. org/10.1109/LISS.2016.7854349.

13. Liu X, Li K, Liu AX, et al. Multi-Category RFID Estimation. IEEE/ACM Transactions on Networking. 2017; 25(1):26477. https://doi.org/10.1109/TNET.2016.2594481.

14. Gibbs A, González E. Implementación de un sistema de control de entrada y salida empleando el módulo de lectura RFID con la tecnología Arduino. Revista de Iniciación Científica. 2017; 2(2):1-16.

15. Gómez JO. Sistema electrónico de control y trazabilidad de medicamentos usando Hardware Arduino con tecnología RFID-RC522. Universidad Tecnologica De Pereira Facultad De Ingenierias Ingenieria Electronica; 2016. p. 1-39.

16. Liu X, Xiao B, Li K. RFID Estimation with Blocker tags. IEEE/ACM Transactions on Networking. 2017; 25(1):22437. https://doi.org/10.1109/TNET.2016.2595571.

17. ElMahgoub K. Pre-encrypted user data for secure passive UHF RFID communication. IEEE, 12th International Computer Engineering Conference (ICENCO); 2016. p. 26-9. https://doi.org/10.1109/ICENCO.2016.7856440.

18. Sarma S, Weis S, Engels D. RFID systems and security and privacy implications. International Workshop on Cryptographic Hardware and Embedded Systems; 2002. p. 454-69. 
19. Dise-o, Desarrollo e Implementación de un Sistema RFIDArduino [Internet]. [cited 2012 Nov 27]. Available from: https://riunet.upv.es/handle/10251/17916.

20. Elías JC. Implementación de WPS en el firmware Node MCU para el ESP8266. (Trabajo fin de grado inédito). Universidad de Sevilla, Sevilla; 2016. p. 1-85. PMCid:PMC5123388

21. Kotiyal B, Muzamil M. Home automation using arduino WiFi module ESP8266. Electronics and Telecommunication; 2016. p. 1-55.
22. Tan C, Sun W, Lu S, Kuo C. P-167: A Simple Architecture for Fully Integrated 2.4 "Poly-Si TFT-LCD. SID Symposium Digest of Technical Papers. 2012; 36(1):1-248.

23. Elmasri R, Navathe S, Castillo V, Pérez G. Sistemas de Información IITema 5. El modelo relacional. Fundamentos de sistemas de bases de datos. 3rd Edition; 2002.

24. Kroenke D, Hernández A. Procesamiento de bases de datos: fundamentos, dise-o e implementación. Pearson Educación, México; 2003. p. 1-488. 